\title{
\begin{tabular}{|l|l|l|l|l|}
\hline $\mathrm{M}$ & $\mathrm{R}$ & $\mathrm{S}$ & Internet Journal of & Nitride Semiconductor Research \\
\hline
\end{tabular}
}

Volume 1, Article 44

\section{Identification of a Cubic Phase in Epitaxial Layers of Predominantly Hexagonal GaN}

\author{
U. Strauss, H. Tews, H. Riechert, R. Averbeck, M. Schienle, B. Jobst \\ Siemens AG, Corporate Research and Development \\ D. Volm, T. Streibl, BK Meyer \\ Physikdepartment, Technische Universitaet Muenchen \\ W. W. Rühle \\ Max-Planck-Institut für Festkörperforschung
}

This article was received on June 3, 1996 and accepted on December 17, 1996.

\begin{abstract}
Epitaxial layers of GaN on c-plane sapphire are analyzed by continuous-wave and time-resolved photoluminescence at $4 \mathrm{~K}$ and by X-ray diffraction. Besides the well-known emissions from hexagonal GaN we observe luminescence bands at 3.279 and 3.15 to $3.21 \mathrm{eV}$ which are identified as the transition of the donor bound exciton and the donor-acceptor pair recombination in cubic GaN, respectively. Measurements of the luminescence decay times are essential for the clarification of the emission processes. Due to the probing depth of about $200 \mathrm{~nm}$ in PL we find that the fraction of cubic phase typically decreases with layer thickness. In our best samples, however, we do not detect the cubic phase at all.
\end{abstract}

\section{Introduction}

$\mathrm{GaN}, \ln _{x} \mathrm{Ga}_{1-x} \mathrm{~N}$, and $\mathrm{Al}_{\mathrm{y}} \mathrm{Ga}_{1-y} \mathrm{~N}$ is suitable for visible light emitting diodes and short-wavelength lasers. [1] [2] [3] There are great efforts to grow epitaxial layers of high quality. For that, there is great interest for characterisation of these layers, for example the crystal perfection. Predominantly, the GaN epitaxial layers crystallise in the most stabile, hexagonal (wurtzite) structure, especially when grown on substrates with hexagonal symmetry such as sapphire or $6 \mathrm{H}-\mathrm{SiC}$. [4] The growth of cubic $\mathrm{GaN}$ was reported for cubic substrates, for example on $\mathrm{GaAs}$, (111)-Si, and (001)-Si with $\beta$-SiC-coating. [5] [6] [7] [8] [9] [10] The presence of both crystal structures was observed in layers on layers on (100)-Si: the predomenantly hexagonal structure contains a cubic minor phase of GaN as detected by $\mathrm{x}$-ray measurements. [9]

In this work, we investigate GaN grown on sapphire (0001) substrates. We examine the presence of a cubic phase in our layers with dominant hexagonal structure. An often used method is an X-ray diffraction measurement (XRD), but XRD detects signals from the whole thickness' of the samples and it is not clear, whether the cubic material is in the top layers or close to the buffer layers. Therefore, we additionally test the use of photoluminescence spectroscopy $(\mathrm{PL})$, which is a method with high sensitivity to the top of the layers up to the penetration depth of about $200 \mathrm{~nm}$. The identification of the luminescence peaks in $\mathrm{GaN}$ is difficult, because there exist many emission processes in GaN with nearly the same emission energies. In this work, the emission peaks of the cubic crystals are identified by time-resolved measurements. We present the structural analysis of the top layer of the GaN samples by using PL.

\section{Experimental}

The investigated layers of GaN were grown by molecular beam epitaxy. A nitrogen microwave plasma source and a solid source of $\mathrm{Ga}$ were used. Thin buffer layers of GaN and the epitaxial GaN layers of approximately $400 \mathrm{~nm}$ thickness were subsequently deposited on (0001) sapphire substrates. The growth temperatures were approximately $700^{\circ} \mathrm{C}$, the growth rates were about $800 \mathrm{~nm} / \mathrm{h}$. Hall measurements characterised the layers as $\mathrm{n}$-type. 
The carrier concentrations range from $5 \times 10^{1 /}$ to $4 \times 10^{18} \mathrm{~cm}^{-3}$ at room temperature. The layers are optically investigated by continuos wave photoluminescence (cw PL), time-resolved photoluminescence (tr PL), and spacially resolved cathodoluminescence ( $\mathrm{sr} \mathrm{CL}$ ), all determinations are carried out at $4.2 \mathrm{~K}$. The $\mathrm{cw} P L$ is measured using a $1 \mathrm{~m}$ spectrometer and a GaAs photomultiplier. For cw PL, the samples are excited with the $325 \mathrm{~nm}$ line of a cw HeCd laser with a spot size of about $0.1 \mathrm{~mm}^{2}$ and $10 \mathrm{~mW}$ power. The tr PL is performed using a $0.25 \mathrm{~m}$ spectrometer and a streak camera with $20 \mathrm{ps}$ time resolution. The laser system for excitation is a frequency-tripled Ti-sapphire laser with an emission of $5 \mathrm{~mW}$ at $265 \mathrm{~nm}$, a repetition rate of $12 \mathrm{MHz}, 100$ fs pulse width, and a spot size of roughly $0.01 \mathrm{~mm}^{2}$. The $\mathrm{sr} C L$ is performed with a voltage of $2 \mathrm{kV}$. The resolution is $1 \mu \mathrm{m}$. We compare our optical data with $\mathrm{x}$-ray diffraction measurements. The $\mathrm{x}$-ray set-up uses the $\mathrm{Cu}-\mathrm{K} \alpha_{1}$-line, the resolution is $0.1^{\circ}$.

\section{Results and Discussion}

The cw PL spectra of three typical kinds of samples are shown in Figure 1. Four dominant emission bands and a weaker emission are observed, labelled with $\underline{A}, \underline{B}, \underline{C}, \underline{D}$, and $\underline{E}$. The luminescence decays of these four emission peaks are listed in the table I. We want to mention that $\mathrm{CW} \mathrm{PL}$, tr PL and sr CL all show the same emission lines.

The origin of the emission $\underline{A}$ can be identified easily as an exciton bound to the residual donor in hexagonal (hex) GaN, labelled as $D_{\text {hex }} 0 X$. This emission line is well known to be dominant in GaN on sapphire. [11] [12] [13] The luminescence decay time of $D_{\text {hex }} 0 X$ is as short as $(50 \pm 20)$ ps due to the well known weak localisation energy. The emission group $\underline{B}$ appears to be related to deeply bound excitons, labelled $B X_{h e x}$. The strong bonding of the excitons is in agreement with the long luminescence decay times of $\underline{B}$ between 500 and 1000 ps.

The emission lines $\underline{\mathrm{C}}$ and $\underline{\mathrm{D}}$ are more difficult to identify. The intensity of $\underline{\mathrm{C}}$ drastically decays with time. The short lifetime of $(30 \pm 20)$ ps is only expected for a shallow bound exciton or recombination of free carriers. We think that $\underline{\mathrm{C}}$ is not a due to the recombination of a free electron with a localised hole, labelled eA. The emission line $\underline{\mathrm{C}}$ is at the same energy like the eA transition in hexagonal GaN, [10] but we do not observe the corresponding DAP transition with a lifetime of several nanoseconds at an emission energy of 0 to $30 \mathrm{meV}$ below $\underline{\mathrm{C}}$. This corresponding DAP transition should even be stronger than the eA transition, because the donor densities are below the degeneration density of $5 \times 10^{18} \mathrm{~cm}^{-3}$ in all of our samples. We conclude, that $\underline{\mathrm{C}}$ is related to a shallow bound exciton. However, there may be a weak contribution of any kind of DAP transition to the emission $\underline{\mathrm{C}}$. The transition energy of $\underline{\mathrm{C}}$ is much to low for a shallow bound exciton in hexagonal GaN, but the energy is typical for cubic (cub) GaN. [10] [14] [15] We

think that $\underline{\mathrm{C}}$ is dominated by the recombination of the donor bound excition in cubic $\mathrm{GaN}$, labelled $\mathrm{D}_{\text {cub }} \mathrm{X}$, since the donor concentrations are higher than the acceptor concentration in undoped GaN.

Next, we want to identify the emission band $\underline{D}$. The lifetime of $\underline{D}$ is as long as $5-20 \mathrm{~ns}$, a value similar to the repetition rate of the pulsed laser. Such a long lifetime is a strong indication of a donor-acceptor pair (DAP) recombination. The position of $\underline{D}$ is different for each sample, an observation which is also typical for DAP transitions. Additional information about the emission peaks is achieved by spacially resolved cathodoluminescence measurements. The major part of our sample shows dominant emission peaks $\underline{A}$ and $\underline{B}$. But in some apparently polycrystalline samples, we observe small areas with diameters below $2 \mu \mathrm{m}$, which show dominant emissions from $\underline{\mathrm{C}}$ and $\underline{\mathrm{D}}$. The emissions $\underline{\mathrm{C}}$ and $\underline{D}$ always appear together and $\underline{C}$ is already identified as an emission from cubic GaN. Therefore, we conclude that $\underline{D}$ is the DAP transition from regions which contain cubic $\mathrm{GaN}$. We have to mention that PLs of deeply bound excitons also would have long decay times. However, the observed shift of the emission energy is typical for DAP transitions and not for bound excitons.

We now look at the band $\underline{E}$. This emission always appears together with $\underline{D}$ and $\underline{E}$ has the same lifetime like $\underline{D}$. The band $E$ is always 90 meV below $D$ and therefore, $E$ is identified as the longitudinal optical phonon replica (LO) of $\underline{D}$. The phonon replicas are weaker than that ones from the DAP transitions in hexagonal GaN. The emission processes and their energies are compiled in the table I. We want to mention that the commony seen yellow luminescence is very week in our samples.

The identification of luminescence from cubic $\mathrm{GaN}$ is confirmed by $\mathrm{x}$-ray diffraction measurements: All samples with luminescence from the cubic phase also show an X-ray diffraction signal of the cubic material. We measure the symmetric peaks in a standard $\theta / 2^{*} \theta$ - diffractometer. One typical measurement is shown in Figure 2 . The $(0004)$ reflection of hexagonal $\mathrm{GaN}$ and the (222) reflection of cubic $\mathrm{GaN}$ are detected. The d-values of the two lattice planes are nearly identical, so that we can distinguish cubic and hexagonal GaN only for reflexes of second and third order. We find the XRD signal of the cubic phase in all samples with the PL peaks $\underline{C}, \underline{D}$ and $\underline{E}$. This correlation between PL and XRD supports our interpretation of the emission processes. Further, there are samples with XRD 
signal of cubic GaN, but the PLs of these samples only show the emissions from hexagonal GaN. We conclude, that the cubic phase is only close to the buffer layer in samples without emissions $\underline{\mathrm{C}}$, $\underline{\mathrm{D}}$ and $\underline{\mathrm{E}}$, the cubic phase extends into the top layer in the samples with the emissions $\underline{C}, \underline{D}$ and $\underline{E}$. This results shows the necessity of PL for the characterisation of the top layers of the samples. From our data, we cannot say wheather our samples contain larger regions of cubic $\mathrm{GaN}$ or wheather there is poly hexagonal material with mis-nucleated grains. But we see that the cubic phase is related to the buffer layer. However, in our best samples, we do not detect a cubic signal at all.

Finally, we now compare our results with earlier data. The emission of the $\mathrm{D}^{0} \mathrm{X}_{\text {hex }}$ is well known and will not be discussed here. Band $\underline{B}$ is similar to an emission observed by Smith et al.. [16] The authors measure and they explain this emission as a recombination of a free hole with an electron at a donor. In our samples, the lifetime if $\underline{B}$ is to long to be related to free holes. The emission at $3.448 \mathrm{eV}$ are similar to acceptor bound excitons in $\mathrm{Zn}$-doped samples. However, to our knowledge there is no $\mathrm{Zn}$ in our MBE chamber.

The luminescence spectra of cubic $\mathrm{GaN}$ usually show two dominant emission peaks, which are similar to $\underline{\mathrm{C}}$ and $\underline{\mathrm{D}}$. [6] [7] [8] [9] [10] [17] Hong et al. measure $90 \mathrm{meV}$ higher emission energies than the other authors, the difference may be related to strain. [17] In the following, we subtract $90 \mathrm{meV}$ from Hong's values to compare all data. The first emission is at about 3.27 eV. Klann et al., [7] Menninger et al., [17] and Hong et al. [8] relate this emission to a bound exciton. In contrast, Hwang et al. assume that there is a eA transition. [7] Liu et al. also relate the emission with the cubic band gap, however, their spectrum is to broad to discuss the mechanism in detail. We relate the emission $\underline{\mathrm{C}}$ to a bound exciton, because the measured temperature dependence of the luminescence is typical for a bound exciton and not for an eA transition. Our identification of $\underline{D}$ as a DAP transition in a cubic minor phase is in agreement with earlier measurements of an emission between 3.15 and $3.21 \mathrm{eV}$ in pure cubic GaN.

\section{Conclusions}

In conclusion, we have demonstrated that photoluminescence measurements reveal the presence of a minor cubic phase in the top $200 \mathrm{~nm}$ of epitaxial layers of hexagonal GaN on sapphire substrates. We have identified the emission of the donor-bound exciton in cubic GaN at an energy of $3.279 \mathrm{eV}$ at $4 \mathrm{~K}$. The emission between 3.15 to $3.21 \mathrm{eV}$ at $4 \mathrm{~K}$ is explained as the donor-acceptor recombination in cubic $\mathrm{GaN}$. We observed a weaker phonon coupling in cubic GaN than in hexagonal GaN.

\section{Acknowledgments}

We like to thank $O$. Brandt for spacially resolved cathodoluminescence measurements and A. Graber for helpful discussions. One of us (U.S.) is a delegate of the Max-Planck-Institut $f_{s} r$ Festk rperforschung. U.S. thanks the Max-Planck-Institut and the German Ministry of Education and Research for their support.

\section{References}

[1] Shuji Nakamura, Takashi Mukai, Masayuki Senoh, Appl. Phys. Lett. 64, 1687-1689 (1994).

[2] I. Akasaki, H. Amano, H. Murakami, M. Sassa, H. Kato, K. Manabe, J. Cryst. Growth 128, 379 (1993).

[3] R. J. Molnar, R. Singh, T. D. Moustakas , Appl. Phys. Lett. 66, 268-270 (1995).

[4] S. Strite, H. Morkoç, J. Vac. Sci. Technol. B 10, 1237-1266 (1992).

[5] C. J. Sun, P. Kung, A. Saxler, H. Ohsato, E. Bigan, M. Razeghi , D. K. Gaskill , J. Appl. Phys. 76, 236-241 (1994).

[6] R. Klann, O. Brandt, H. Yang, H. T. Grahn, K. Ploog , A. Trampert, Phys. Rev. B 52, 11615-11618 (1995).

[7] S. J. Hwang, W. Shan, R. J. Hauenstein, J. J. Song , M.-E. Lin, S. Strite, B. N. Sverdlov, H. Morkoc , Appl. Phys. Lett. 64, 2928-2930 (1994).

[8] C. H. Hong, D. Pavlidis, S. W. Brown, S. C. Rand, J. Appl. Phys. 77, 1705-1709 (1995). 
[9] T. D. Moustakas, T. Lei, R. J. Molnar, Physica B 185, 36-49 (1993).

[10] J. G. Kim, A. C. Frenkel, H. Liu, R. M. Park, Appl. Phys. Lett. 65, 91-93 (1994).

[11] R. Dingle, M. llegems, Sol. St. Comm. 9, 175 (1971).

[12]S. Fischer, C. Wetzel, W. L. Hansen, E. Bounet, and E. E. Haller, unpublished.

[13] B. K. Meyer, D. Volm, A. Graber, H. C. Alt, T. Detchprohm, A. Amano, I. Akasaki, Sol. St. Comm. 95, 597 (1995).

[14] H. Okumura, S. Yoshida, T. Okahisa, Appl. Phys. Lett. 64, 2997-2999 (1994).

[15] G. Ramirez-Flores, H. Navarro-Contreras, A. Lastras-Martinez , R. C. Powell, J. E. Greene , Phys. Rev. B 50, 8433-8438 (1994).

[16] M. Smith, G. D. Chen, J. Y. Lin, H. X. Jiang , A. Salvador, B. N. Sverdlov, A. Botchkarev, H. Morkoc, Appl. Phys. Lett. 66, 3474-3476 (1995).

[17] J. Menniger, U. Jahn, O. Brandt, H. Yang, K. Ploog , Phys. Rev. B 53, 1881-1885 (1996).

\section{Table I}

The emission energies of the luminescence peaks at $4.2 \mathrm{~K}$ and the identification of the emission processes.

label PL energy (eV) PL decay time (ps) identified as
A $\quad 3.472$
$50 \pm 10$
$\mathrm{D}_{0} \mathrm{X}_{\text {hex }}$
B $\quad 3.424-3.448 \quad 500$ to 1000
$B X_{\text {hex }}$
C $\quad 3.279$
$30 \pm 20$
$\mathrm{D}_{0} \mathrm{X}_{\text {cub }}$
D7 $3.15-3.21$
5000 to 20000
DAP $_{\text {cub }}$
E $\quad 3.06-3.12$
same like DAP
DAP $_{\text {cub }}$ - LO

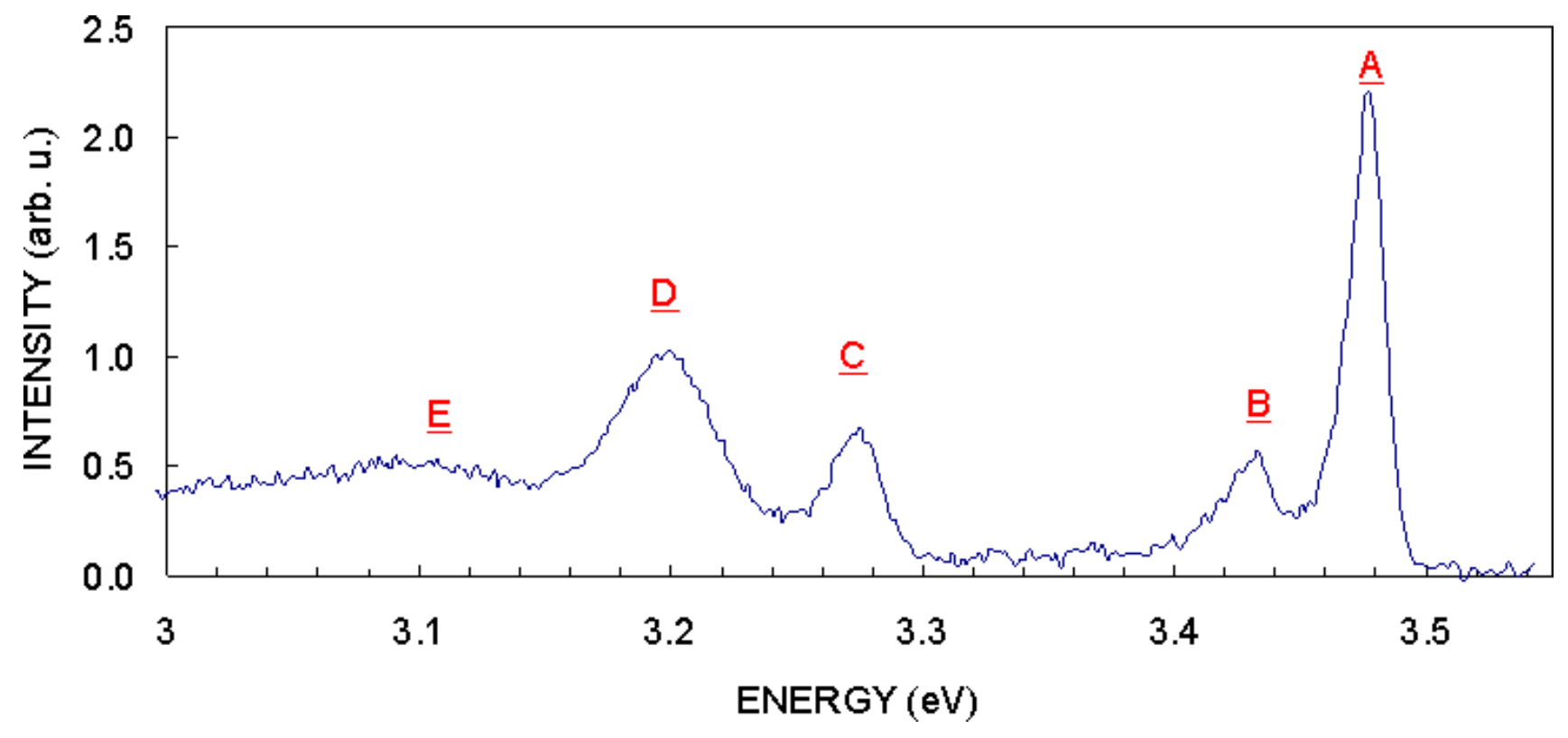

Figure 1. Photoluminescence spectrum of epitaxial GaN. The sample is selected from a series of samples with strong emission between 3.1 and $3.3 \mathrm{eV}$. 


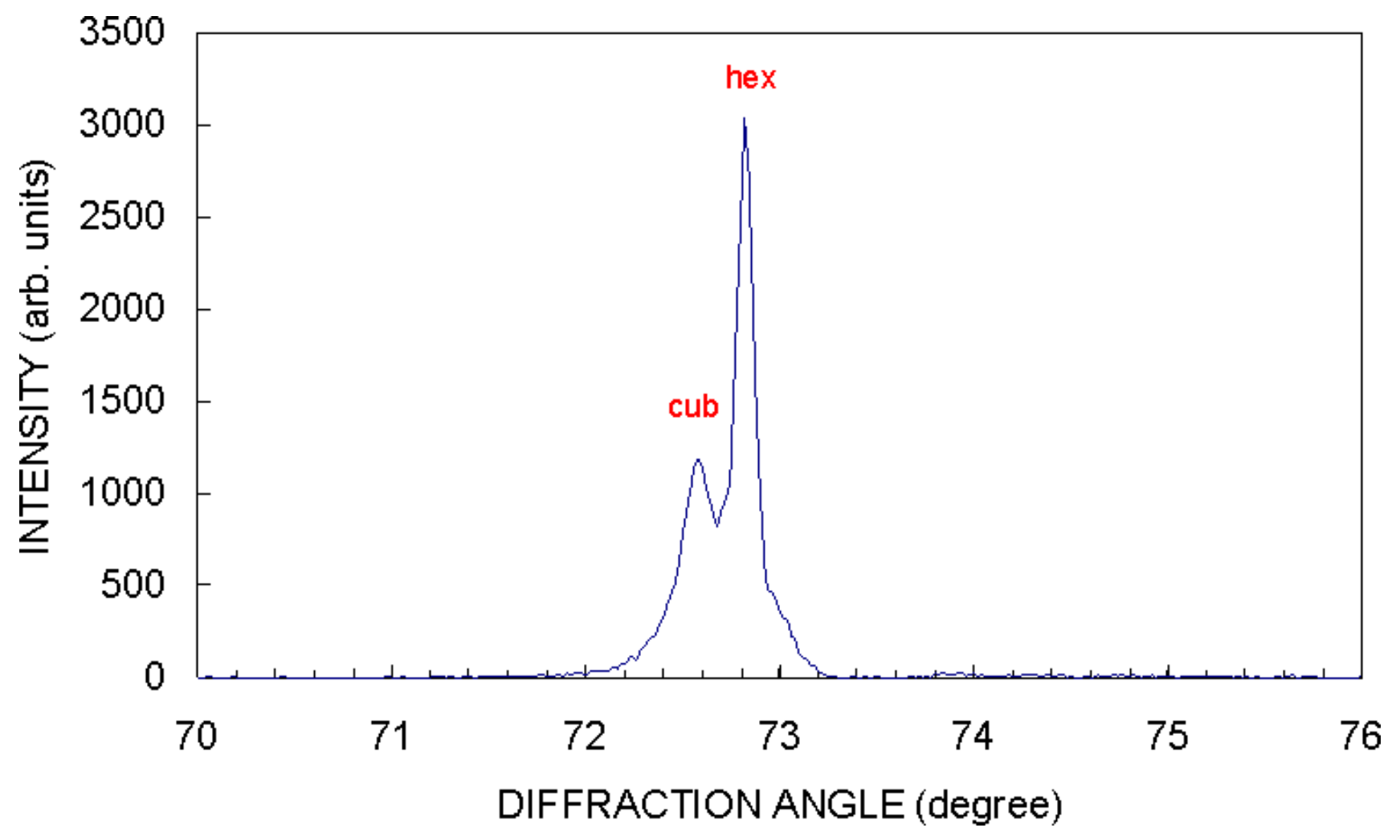

Figure 2. The $\theta-2 \theta$ scan of one sample which contains the cubic and hexagonal phase. The two peaks are identified as the (222) reflexion of cubic GaN and the (0004) reflexion of hexagonal GaN, respectively.

(C) 1996-1998 The Materials Research Society

\begin{tabular}{|l|l|l|l|l|}
\hline $\mathrm{M}$ & $\mathrm{R}$ & $\mathrm{S}$ & Internet Journal of & Nitride Semiconductor Research \\
\hline
\end{tabular}

